\title{
Bilataral Peripheral Gangrene Following Malaria Parasitaemia At Butare University Hospital - A Case Report.
}

\author{
Ahmed Kiswezi Kazigo, \\ Department of Surgery, Butare University Teaching Hospital, Rwanda. \\ Correspondence to: Email: ahmedkiswezi@gmail.com, \\ https://dx.doi.org/10.4314/ecajs.v22i1.15
}

In many parts of the East African region malaria is endemic, while in other parts it is hyper-endemic. While all the four species of the plasmodium parasite (vivax, ovale, malaria\& falciparum), are prevalent in E Africa, it is the Plasmodium falciparum that is most aggressive and rampant. In this region malaria is still by far among the top three disease burdens accounting for high morbidity and mortality rates across all age groups. Whereas there are many complications associated with severe malaria, peripheral gangrene leading to amputation of limbs is so rare and unbelievable. The following is a summarized report of two cases with bilateral peripheral gangrene seen at Butare University Teaching Hospital, following severe malaria between August and October 2015. In both cases several investigations were carried out to rule out other possible causes of limb ischemia and gangrene.

Key words: Peripheral gangrene; Plasmodium falciparum parasitaemia; Amputation

Case 1

N.S was a 30 year old male who was first admitted at a District hospital with a febrile illness, two weeks prior to transfer to Butare University Teaching Hospital. The reason for transfer was the development of gangrene of both feet. The referral notes indicate that he was admitted at the district hospital with a high fever, with temperatures between $38-40$ degrees Celsius. The blood film showed Plasmodium falciparum malaria (+++) scizont forms. While still on treatment with coartem, his hemoglobin fell to $5 \mathrm{mg} / \mathrm{dl}$ and was given blood transfusion. A few days later he developed pain and numbness of the feet. These latter symptoms became progressive even when fever subsided. He was referred on the tenth day from the District hospital when his feet had developed a dark discoloration.

At Butare U.T.H, the main complaint was darkening, drying, and numbness of his feet. Prior to this febrile illness, he had been in good health, with no history suggestive of diabetes mellitus, cardiovascular disease, or any claudication. He had never smoked cigarettes, and there was no history of trauma. He was not febrile but had dry gangrene of both feet. The popliteal and femoral pulses were of good volume.The following investigations were done: 


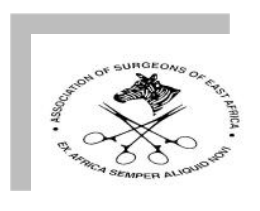

Doppler ultrasound, blood sugar, lipid profile, ECG and ECHO-cardiography, clotting profile, full blood count. All the results were normal, except the Doppler ultra sound, which reported as follows:

A increased resistance to blood flow in the lower $1 / 3$ of the right and left anterior tibial arteries, and almost complete obstruction to flow in the distal $1 / 3$ of the posterior tibial arteries. The middle and proximal parts of the arteries showed normal flow.

After waiting for demarcation of the gangrene, bilateral below knee amputation was done. He was discharged later in a wheel chair.

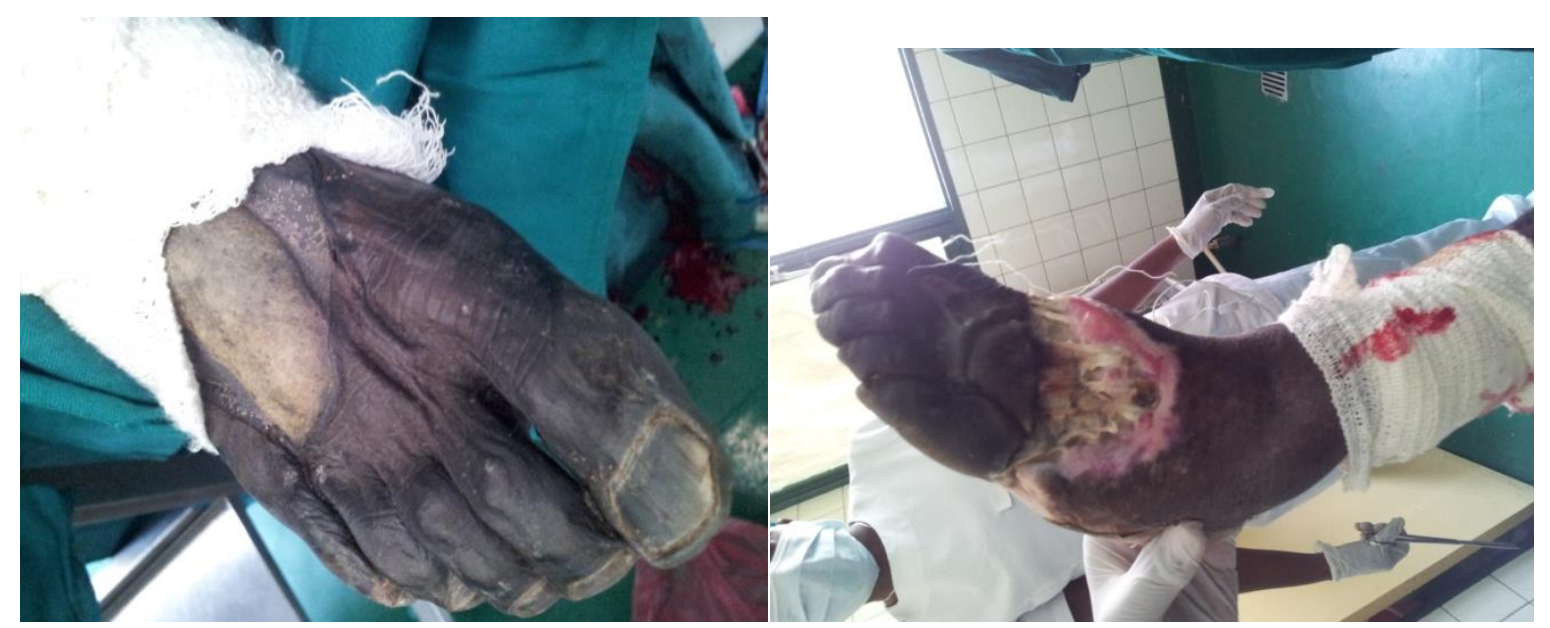

Fig 1: 30 year old N.S had below knee amputation following severe malaria with a high parasitaemia of Plasmodium falciparum.

Case 2

MJP 8 years old male, was referred from a district hospital for further management of bilateral gangrene of all his toes, and the tip of the left small finger. This followed a severe febrile illness characterized by high grade fever, seizures, and coma. With a blood film demonstrating Plasmodium falciparum +++ (gametocytes and ring forms), he was treated for cerebral malaria for six days prior to the gangrene. He was treated with intravenous Quinine. There was no history suggestive of cardiovascular disease, or diabetes. We carried out several investigations including ECG/ECHO, blood sugar, full blood count, and Doppler ultrasound.

All results were normal except the Doppler Ultrasound report which suggested that there was greatly reduced flow in the middle and distal $1 / 3$ of the right and left t posterior tibial arteries; and much reduced flow in both the right and left distal $1 / 3$ of the anterior tibial arteries. Both dorsalis pedis arteries demonstrated disappearing signals. Haematologically, he had a hemoglobin of $7 \mathrm{gm} / \mathrm{dl}$, and there was evidence of recent disseminated intravascular coagulopathy (DIC). While waiting for demarcation, the gangrene spread, involving the mid $1 / 3$ of his feet. He later underwent chopart's (mediotarsal) amputation. 

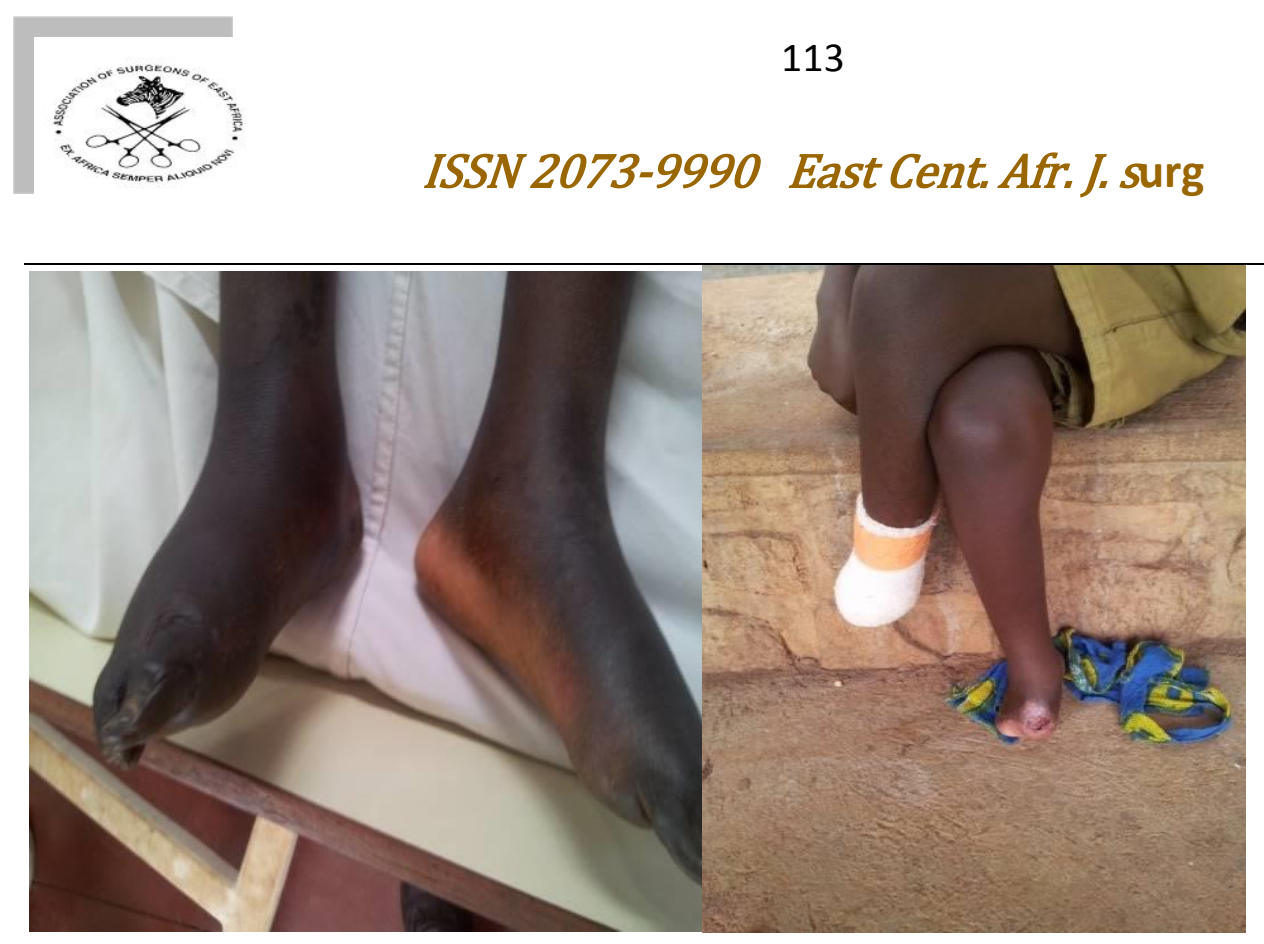

Figure 2.MJP 8 years old, first underwent chopart amputation for bilateral peripheral gangrene of his feet following cerebral malaria with high parasitaemia of Plasmodium falciparum. Later, the left limb developed osteomyelitis of the stump, resulting into further debridement.

\section{Literature Review:}

There is evidence that patients with Plasmodium falciparum malaria may present with peripheral gangrene as a rare complication. Two adult Sudanese patients with high grade fever due to P. falciparum malaria developed bilateral feet gangrene (Saif Eldin Mohammed Ali, Khartoum, 2011)

A 30 year old Ethiopian female developed spontaneous gangrene of the distal phalanges of the index and middle fingers, plus the great and middle toes of the right foot, following cerebral malaria.( Yegerem Kabede, Addis Ababa, 1998)

\section{Discussion:}

These two patients presented with bilateral peripheral gangrene following severe febrile illnesses during which high parasitaemias of Plasmodium Falciparum were hematologically demonstrated.Several investigations were carried out in both cases to exclude other possible causes of gangrene such as diabetes, vascular diseases, cardiac diseases, and localized sepsis.

The pathogenesis of the gangrene in these cases is explained by the fact that high parasitaemia, often a characteristic of plasmodium falciparum, is associated with vascular occlusion, facilitated by a big number of infected RBCs that get adherent to the vascular endothelium, and as the blood vessels become smaller towards the periphery, the risk of vascular occlusion increases. The high parasitaemia is also thought to cause activation of 
complement, leading to thrombosis. In severe malaria, inflammatory cytokines such as TNF and interleukin 6 increase tissue factor interaction with molecular cells, leading to thrombosis. The other possible explanations in these cases may have been the increased viscosity of blood that may be partly due to the rosetting of the infected and non-infected erythrocytes, and DIC.

Gangrene as a complication of malaria has been so rare that it is almost unbelievable in the East African region, despite the high prevalence of malaria. The fact that two cases occurring in the same province were seen in a period of two months, may possibly suggest two things; an up-surge of malaria prevalence, and the changing dynamics of the complication pattern of the disease. But generally, there were several delays in the case management of these patients that could have contributed to the complications. The patients should have been referred for severe malaria, long before they developed gangrene.

The lessons to learn from this include the need for increase in prevention measures, early and vigorous case-management of malaria, with the involvement of community based health workers; and early referral when there are complications.

\section{References}

1. Malaria-associated peripheral gangrene; Deborah B Martins, Journal of Pediatric Surgery, 2014 volume 2

2. Severe Plasmodium Falciparum malaria and peripheral gangrene. Ibrahim SMA, Harunarashid. UKM Journal 2011.

3. Peripheral gangrene following cerebral malaria. Yegremu Kabede et al Addis Ababa. Bioline .Org

4. Edwards IR. Malaria with disseminated intravascular coagulation and peripheral tissue necrosis successfully treated with streptokinase. Br Med J 1980;

280(6226):1252-1253 